\title{
Comparison of diagnostics for the detection of asymptomatic Plasmodium falciparum infections to inform control and elimination strategies
}

\author{
Lindsey Wu*1, Lotus L. van den Hoogen*1, Hannah Slater², Patrick G. T. Walker², Azra C. Ghani², \\ Chris J. Drakeley ${ }^{1} \&$ Lucy C. Okell ${ }^{2}$
}

The global burden of malaria has been substantially reduced over the past two decades. Future efforts to reduce malaria further will require moving beyond the treatment of clinical infections to targeting malaria transmission more broadly in the community. As such, the accurate identification of asymptomatic human infections, which can sustain a large proportion of transmission, is becoming a vital component of control and elimination programmes. We determined the relationship across common diagnostics used to measure malaria prevalence - polymerase chain reaction (PCR), rapid diagnostic test and microscopy - for the detection of Plasmodium falciparum infections in endemic populations based on a pooled analysis of cross-sectional data. We included data from more than 170,000 individuals comparing the detection by rapid diagnostic test and microscopy, and 30,000 for detection by rapid diagnostic test and PCR. The analysis showed that, on average, rapid diagnostic tests detected $41 \%(95 \%$ confidence interval $=26-66 \%$ ) of PCR-positive infections. Data for the comparison of rapid diagnostic test to PCR detection at high transmission intensity and in adults were sparse. Prevalence measured by rapid diagnostic test and microscopy was comparable, although rapid diagnostic test detected slightly more infections than microscopy. On average, microscopy captured $87 \%$ (95\% confidence interval $=74-102 \%)$ of rapid diagnostic test-positive infections. The extent to which higher rapid diagnostic test detection reflects increased sensitivity, lack of specificity or both, is unclear. Once the contribution of asymptomatic individuals to the infectious reservoir is better defined, future analyses should ideally establish optimal detection limits of new diagnostics for use in control and elimination strategies.

Nature 528, S86-S93 (3 December 2015), DOI: 10.1038/nature16039

This article has not been written or reviewed by Nature editors. Nature accepts no responsibility for the accuracy of the information provided.

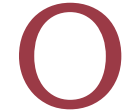

ver the past two decades, considerable progress has been made in reducing the global malaria burden. Between 2000 and 2013 alone, malaria-related mortality decreased by $47 \%$ worldwide and $54 \%$ in Africa. In addition, more than half of malaria endemic countries are on track to meet global targets to reduce malaria incidence by $75 \%$ in 2015 (ref. 1). These achievements are largely due to the widespread use of insecticide-treated nets (ITNs) and highly effective antimalarial treatments. The treatment of symptomatic cases in particular has been enabled by notable advances in the development and deployment of more accurate malaria diagnostics ${ }^{2,3}$. However, efforts to reduce the burden of malaria infections further in the future will require moving beyond the treatment of clinical infections to targeting transmission more broadly in the community. As such, the accurate identification of asymptomatic human infections, which can sustain a large proportion of transmission, is becoming a vital component of control and elimination programmes ${ }^{2,4}$.

Community chemotherapy (for example, mass screen and treat (MSAT) or mass drug administration (MDA) programmes) in conjunction with ongoing vector control is an approach under consideration for the interruption of transmission. This is achieved through the direct treatment of potentially infectious individuals. In the case of MSAT strategies, delivering drugs specifically on the basis of positive test results may be considered preferable to presumptive treatment because it provides clear benefit to the recipient and limits excess drug use that may drive antimalarial resistance. However, owing to the insufficient sensitivity of existing field diagnostics used to identify asymptomatic infections, studies have shown that MSAT has limited effect in reducing transmission ${ }^{5,6}$.

Measuring parasite infection by microscopy has been the gold standard in malaria research for more than a century and remains relatively widespread as a point-of-care diagnostic in clinical and epidemiological settings. More recently, the advent of rapid diagnostic tests (RDTs), which measure the presence of histidine-rich protein 2 (HRP2) for Plasmodium falciparum and/or lactate dehydrogenase for other Plasmodium species ( $p L D H)$, has expanded the range of diagnostic options. Originally developed to inform clinical treatment, RDTs are increasingly important for epidemiological characterization ${ }^{7}$ because of their low cost and field applicability. However, most only have reported detection limits in the range of 100 to 200 parasites per microlitre ${ }^{8,9}$ in comparison with around 50 parasites per microlitre by expert microscopy ${ }^{10}$.

Over the past three decades, the development of nucleic acid amplification tests has improved the detection limit for malaria infection to less than 1 parasite per microlitre by ultrasensitive quantitative polymerase chain reaction $(\mathrm{qPCR})^{11,12}$. Although these detection thresholds are more appropriate for

*These authors contributed equally. ${ }^{1}$ Department of Immunology and Infection, Faculty of Infectious and Tropical Diseases, London School of Hygiene and Tropical Medicine, Keppel Street, London WC1E 7HT, UK. ${ }^{2}$ MRC Centre for Outbreak Analysis and Modelling, Department of Infectious Disease Epidemiology, Faculty of Medicine, Imperial College London, Norfolk Place, London W2 1PG, UK. Correspondence should be addressed to: L. W. e-mail lindsey.wu@lshtm.ac.uk. 


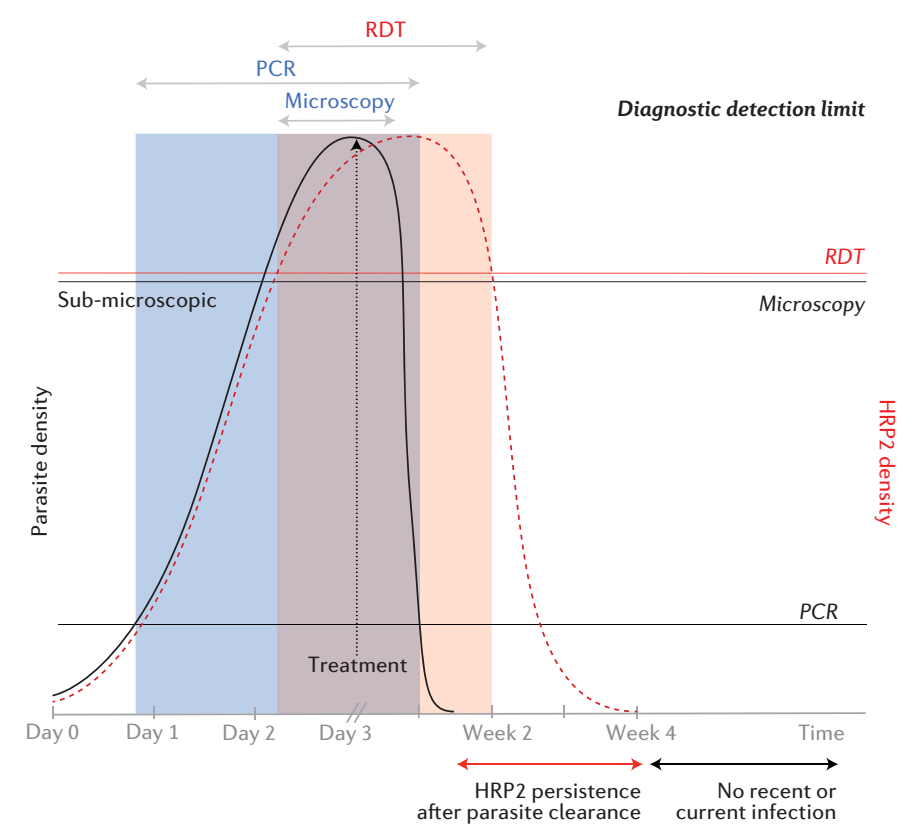

Figure 1|Schematic of diagnostic detection limits with respect to parasite and HRP2 density. The black curve indicates parasite density and the red curve indicates HRP2 density. Time scale is in days prior to treatment and in weeks after treatment. Horizontal lines are the detection limits of respective diagnostics. The blue shaded area shows detectability of parasites by microscopy and/or polymerase chain reaction (PCR), whereas the red shaded area shows detectability of HRP2 by rapid diagnostic test (RDT).

measuring low-density infections than microscopy and RDTs, most PCR techniques remain impractical for wide-scale use in field surveys owing to cost, processing time and the lack of appropriate laboratory facilities in many endemic countries ${ }^{10}$. Comparative analysis of malaria prevalence, measured by both microscopy and PCR in cross-sectional surveys, has shown that sub-microscopic low-density infections are common across a range of transmission settings ${ }^{13,14}$. These infections may be chronic and asymptomatic, particularly in previously exposed individuals with more mature immune responses. More importantly, even at low parasite densities, they are still capable of infecting mosquitoes and seeding onward transmission ${ }^{15}$. Even though RDTs are becoming more common in areas where these types of infections are prevalent, studies formally evaluating their performance in detecting asymptomatic infections remain scarce.

Recently, there has been an increased focus on developing improved diagnostics to inform malaria elimination strategies. The analysis presented in this paper aims to determine the concordance of current malaria diagnostic methods, forming a baseline to evaluate further how they can be improved to inform malaria control and elimination strategies. It should be noted that, in principle, quantifying the presence of gametocytes is considered the most accurate method for characterizing transmission and the potential infectiousness of individuals. Research in this area is ongoing, but the technical challenges of existing gametocyte assays preclude them from standardized use ${ }^{16}$. Moreover, all malaria infections have the capacity to produce gametocytes ${ }^{17,18}$. Therefore, in the context of community chemotherapy programmes, any individual who tests positive for asexual parasites should be treated to reduce transmission. Given this operational framework, this paper does not address the role of diagnostics that specifically measure gametocytaemia.

So far, no studies have comprehensively evaluated the concordance across PCR, RDT and microscopy detection methods simultaneously in asymptomatic populations. Although microscopy- and PCR-measured prevalences are based on similar biological endpoints (parasite density), diagnostic results based on RDTs are less comparable given that HRP2 and pLDH are indirect measures of parasite biomass ${ }^{19}$. HRP2 can persist in the blood for up to two weeks after parasite clearance ${ }^{20}$. Consequently, results across these diagnostic methods indicate a range of possible infection states, from patent or sub-microscopic infection to recently cleared infection (Fig. 1). A limited number of studies have reviewed the detection capability of RDTs in asymptomatic individuals ${ }^{8,21}$, but key research questions still remain. A recent analysis of Demographic and Health Surveys (DHS) across Africa showed a higher prevalence of malaria when measured by RDTs compared with detection by microscopy in 19 out of 22 surveys. This report also highlighted the issue of false positives owing to prolonged presence of HRP2 after parasite clearance ${ }^{21}$. However, studies have not reviewed the detection capability across all three diagnostics. Furthermore, the DHS study only considered children under 5 years of age and did not determine the effect of malaria transmission intensity on diagnostic discordance. This is particularly important given that low-density infections seem to be most common in adults and in low-transmission settings s,14. $^{13}$.

In this study, we determine the relationship across malaria prevalence measures obtained by current diagnostic methods - PCR, RDT and microscopy - for the detection of $P$. falciparum infections in endemic populations based on a pooled analysis of published and unpublished cross-sectional data.

\section{METHODS}

Literature review and data collection. We carried out two separate literature reviews to identify studies in which $P$. falciparum prevalence was measured by different diagnostic techniques in the same individuals: first, by RDT and microscopy, and, second, by RDT and PCR. Relevant studies were identified in PubMed and Embase, using MeSH and Map terms when possible. For the RDT and microscopy review, the search terms were: "'rapid diagnostic test' and 'microscopy' [MeSH/Map] and 'malaria falciparum' [MeSH/Map]", and for the RDT and PCR review the search terms were: "'polymerase chain reaction' [MeSH/Map] and 'malaria falciparum' [MeSH/Map]". Searches were limited to English, human and post-2005 (considering the substantial development in RDTs over time ${ }^{22}$ ). For Embase, the searches were also limited to journal articles. Inclusion criteria were applied as previously described ${ }^{13}$. In short, only studies that were cross-sectional (on populations not selected according to malaria test results or symptoms), that were of populations from a malaria endemic region, that used RDTs targeting $P$. falciparum only or mixed infections (HRP2 and/or pLDH) and that used PCR or loop-mediated isothermal amplification (LAMP) methods were included. For intervention studies, only baseline data were included, except for treatment studies where a sufficient amount of time had passed between last treatment and follow-up. Separate publications that used the same data set or measured $0 \%$ prevalence by both methods were removed, as well as data from clusters with fewer than five individuals. RDT and microscopy studies identified in our literature search that also included PCR measurements were included in the RDT and PCR data set, and vice versa for RDT and PCR studies that included microscopy measurements. In addition to the literature review, we sought as many individual-level data sets as possible from studies with the above inclusion criteria.

RDT and microscopy. Where available, information on location, sample size, RDT brand and type (HRP2 or pLDH), age group (15 or younger compared with older than 15) and prevalence estimates were recorded ${ }^{5,23-42}$. Furthermore, data from the DHS online database were extracted ${ }^{43}$. These included individual-level data on location and timing of collection, RDT and microscopy test results, RDT brand ${ }^{21}$, age, sex, use of an ITN, fever and antimalarial use in the past two weeks. In addition, individual-level data sets from one unpublished and one published study were included ${ }^{44}$, as well as shared data sets of the RDT and PCR comparison that also included microscopy measurements (see below) ${ }^{45-49}$.

RDT and PCR. Corresponding authors of the 13 studies identified from the literature search were contacted to request individual-level data in December 2014 and reminders were sent out 4 weeks later. Of the contacted authors, six responded within the timeframe; five data sets were included ${ }^{45-47,49,50}$, and one data set had been destroyed for privacy compliance. Prevalence measures and study information (including PCR method) were extracted as described above from the publications in the aforementioned literature search and the non-responders group, as well as included studies from the RDT and microscopy search that also reported PCR proportions ${ }^{25,27,34,39,40,42,51-55}$. Four additional individual-level unpublished and published data sets were included 44,48 . 
Statistical analyses. We analysed the association between PCR- and RDT-measured prevalence, and microscopy- and RDT-measured prevalence by fitting a linear relationship on the log odds scale $e^{13,56}$. Prevalence (on a scale of 0 to 1) was defined as $\frac{e^{(\log o d d s)}}{\left.1+e^{(\log \text { odd } s}\right)}$, where log odds $=\log _{\mathrm{e}}\left(\frac{\text { prevalence }}{1-\text { prevalence }}\right)$

$\Omega_{R i}=\Omega_{P i}+\delta_{R i}$

$\delta_{R i}=\delta_{R i}^{\prime}+\beta_{0}\left(\Omega_{P i}-\bar{\Omega}_{P}\right)$

$\Omega_{R i}=\Omega_{M i}+\delta_{R i}$

$\delta_{R i}=\delta_{R i}^{\prime}+\beta_{0}\left(\Omega_{M i}-\bar{\Omega}_{M}\right)$

In Equations 1-4, $\Omega_{R i}$ is the log odds of RDT-measured prevalence in trial $i$, $\Omega_{P i}$ is the log odds of PCR prevalence, $\Omega_{M i}$ is the log odds of microscopy-measured prevalence, $\delta_{R i}$ is the log odds ratio (OR) of RDT- to PCR-measured prevalence (RDT:PCR; Equation 1) or RDT- to microscopy-measured prevalence (RDT:microscopy; Equation 3), $\delta_{R i}^{\prime}$ is the expected log OR of RDT:PCR prevalence (Equation 2) or RDT:microscopy prevalence (Equation 4) when the log odds of PCR- or microscopy-measured prevalence is equal to the mean across trials, $\bar{\Omega}_{P}$ and $\bar{\Omega}_{M}$ are the mean log odds of PCR- and microscopy-measured prevalence, respectively, across trials, and $\beta_{0}$ is the regression coefficient. To allow for varying sample size and sampling variation across the surveys included in our analysis, the model was fitted using Bayesian Markov Chain Monte Carlo methods in JAGS version 3.4.0 and the rjags package in $\mathrm{R}$ version 3.0.2 (ref. 13). We also explored fitting polynomial relationships, but these provided no substantial improvement in fit to the data over the linear model as assessed by deviance information criterion, nor were these fitted relationships qualitatively different (data not shown). To confirm that the fitted curves at different prevalence ranges were not overly influenced by the high number of data points in lower transmission areas, we fitted separate relationships in three PCR-measured prevalence bands: $<5 \%, 5-20 \%$ and $>20 \%$. These categories represent approximate cut-offs that have been suggested as thresholds for operational decision-making. Broadly speaking, programmes can begin to consider targeted and focal control strategies when parasite prevalence by microscopy falls below $5 \%$ (ref. 57), which translates to a PCR-measured prevalence of $20 \%$ (ref. 14), and move towards targeted elimination when it falls below $1 \%$ (ref. 58) (5\% PCR-measured prevalence ${ }^{14}$ ).

We also conducted a meta-analysis of the risk ratio between RDT:PCR prevalence or RDT:microscopy prevalence, adjusted for random effects at the study level (for RDT:PCR) or country level (for RDT:microscopy). Studies that reported zero infections by either diagnostic method were assigned a value of 0.01 to allow a risk ratio to be calculated. To evaluate the effect of explanatory factors on discordant test results, individual-level data were analysed by logistic regression, allowing for random effects at the study or country level as noted above. The meta-analysis was done with the metafor package in $\mathrm{R}$ version 3.0.2, and the logistic regression with the logit command in STATA version 13.

We assessed the ability of our models to predict RDT-measured prevalence based on microscopy- or PCR-measured prevalence data. Leave-one-out cross validation was used to evaluate the RDT:PCR and the RDT:microscopy models separately. The data available for direct comparison of malaria detection by RDT and PCR in the same individuals were sparse relative to the quantity of data available for the RDT:microscopy and previous microscopy:PCR comparisons. Therefore, we also triangulated the relationship between RDTand PCR-measured prevalence by combining the RDT:microscopy relationship calculated in this study with the microscopy:PCR prevalence relationship that has been previously defined ${ }^{13}$. The credible interval of the triangulation line was computed from the posterior distributions of all the parameters from both equations combined. We evaluated whether this triangulated RDT:PCR relationship was significantly different from the observed RDT:PCR relationship using the posterior distributions of the predictions from each model.

\section{RESULTS}

Literature search and data collection. The literature search generated 549 results in Pubmed and an additional 37 in Embase for RDT and microscopy, and 2,247 results in PubMed and an additional 426 in Embase for RDT and PCR. In
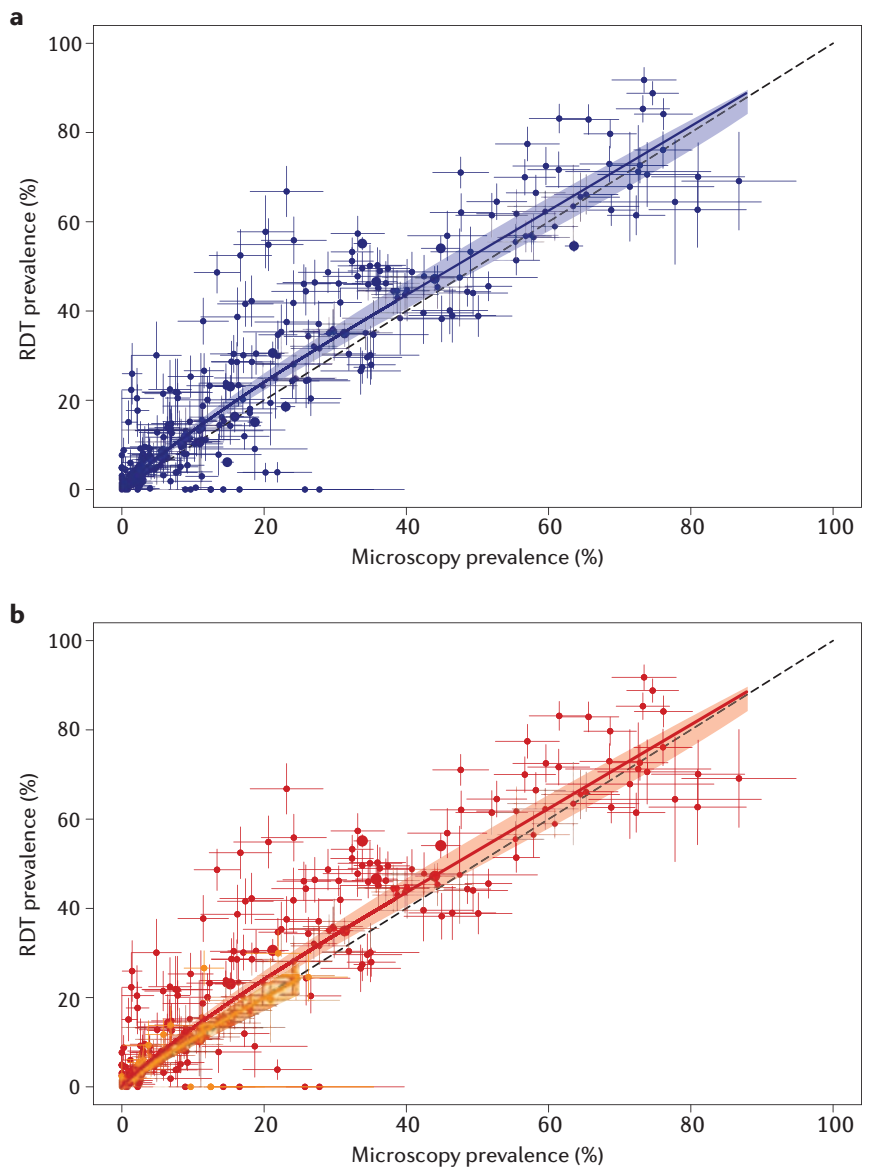

Figure 2 | The relationship between rapid diagnostic tests (RDTs) and microscopy Plasmodium falciparum prevalence overall (a) and stratified by age group (b). In $\mathbf{b}$ red indicates children (those under 15 years) and yellow indicates adults (those over 15 years). Dashed lines indicate the expected relationship if RDT and microscopy detected equal prevalence. Horizontal and vertical lines indicate $95 \%$ confidence intervals around point estimates, whereas coloured solid lines indicate the median of the Bayesian posterior distributions from the fitted model and shaded areas indicate $95 \%$ credible intervals. Radius of point estimates indicate cluster size (from small to large: $<100,100-1,000$ and $>1,000$ ).

total, 20 RDT: microscopy studies and 13 RDT:PCR studies from the literature search met our inclusion criteria. Combined with additional data sets from DHS and unpublished studies, the pooled data available for evaluation yielded 323 pairs of prevalence estimates for RDT and microscopy ${ }^{5,23-42,44-49}$ and 162 pairs for RDT and PCR $25,27,34,39,40,42,45-55$. The extracted proportions together with the main characteristics of the studies from our literature search are provided in the Supplementary Information. The main PCR method used was nested PCR ( $n P C R ; 15$ of 20) of which mainly the Snounou method ${ }^{59}$ was used (11 of 15). The other methods included LAMP ( 1 of 20 ) and APCR ( 4 of 20). All of the included RDTs in both comparisons were based on HRP2, with 8 out of 20 studies also including pLDH to measure species other than P. falciparum. However, this study only focuses on the detection of $P$. falciparum infections.

Comparison of RDT- and microscopy-measured prevalence. Analysis of RDT- and microscopy-measured prevalence included data from 172,281 individuals who were tested with RDTs (cluster prevalence range $=0-92 \%$ ) and 186,434 tested with microscopy (cluster prevalence range $=0-87 \%$ ). The 323 geographical clusters spanned a total of 29 countries (cluster size range $=$ $5-7,664)$. Overall, prevalence of $P$. falciparum measured by microscopy detected $87 \%$ ( $95 \%$ confidence interval $(\mathrm{Cl})=74-102 \%$ ) of RDT-positive infections. Therefore, RDT and microscopy detection was comparable (Fig. 2a, Table 1), with less of a difference between the two diagnostic methods in children under 15 years of age $(77 \%, 95 \% \mathrm{Cl}=71-85 \%)$ compared with adults (over 15 years) 
Table 1 | Best fit relationships between RDT:microscopy and RDT:PCR prevalence.

\begin{tabular}{|c|}
\hline RDT:microscopy \\
\hline OVERALL \\
\hline log odds RDT prevalence $=0.108+0.907 \times \log$ odds microscopy prevalence (all ages) \\
\hline BY AGE CATEGORY \\
\hline log odds RDT prevalence $=0.109+0.908 \times \log$ odds microscopy prevalence (under 15 years) \\
\hline log odds RDT prevalence $=-0.168+0.890 \times \log$ odds microscopy prevalence (over 15 years) \\
\hline RDT:PCR \\
\hline OVERALL \\
\hline log odds RDT prevalence $=-0.968+1.186 \times \log$ odds PCR prevalence (all ages) \\
\hline BY AGE CATEGORY \\
\hline log odds RDT prevalence $=-0.382+1.306 \times \log$ odds PCR prevalence (under 5 years) \\
\hline log odds RDT prevalence $=-0.864+1.213 \times \log$ odds PCR prevalence $(6-15$ years $)$ \\
\hline log odds RDT prevalence $=-1.378+1.300 \times \log$ odds PCR prevalence (over 15 years) \\
\hline BY AGE CATEGORY \\
\hline log odds RDT prevalence $=1.097+1.690 \times \log$ odds PCR prevalence $(<5 \%$ prevalence $)$ \\
\hline log odds RDT prevalence = $0.211+1.754 \times$ log odds PCR prevalence $(5-20 \%$ prevalence $)$ \\
\hline log odds RDT prevalence $=-0.516+1.904 \times \log$ odds PCR prevalence $(>20 \%$ prevalence $)$ \\
\hline PCR PREVALENCE BASED ON DATA TRIANGULATION \\
\hline log odds PCR prevalence $=0.108+0.907 \times[$ [log odds RDT prevalence -0.954$) / 0.868]$ \\
\hline$P C R$, polymerase chain reaction; $R D T$, rapid diagnostic test. \\
\hline $\begin{array}{l}(60 \%, 95 \% \mathrm{Cl}=48-86 \%) \text { (Fig. } 2 \mathrm{~b} \text {, Table } 1) \text {. The lower age-specific risk ratios } \\
\text { are due to smaller cluster sizes after stratifying the data by age group. However, } \\
\text { regression analysis of individual-level data did not show a significant associa- } \\
\text { tion between age group and test discordance (Supplementary Table 1). }\end{array}$ \\
\hline
\end{tabular}

Effect of individual level covariates on RDT:microscopy discordance. In addition to age, we explored the effect of several other covariates on diagnostic outcomes, and adjusted for transmission intensity as assessed by microscopy-measured prevalence (Supplementary Table 1). A significant association was seen between self-reported antimalarial use in the two weeks before survey testing and RDT positivity in individuals who tested negative by microscopy $(O R=1.71,95 \% \mathrm{Cl}=1.16-2.51, p=0.006)$. The presence of fever at the time of testing (recorded temperature with study-specific cut-off or self-reported) reduced the odds of undetected malaria infection by RDT among microscopy-positive individuals $(\mathrm{OR}=0.59,95 \% \mathrm{Cl}=0.39-0.89$, $p<0.001)$. Among individuals testing negative by microscopy, presence of a fever was significantly associated with RDT positivity $(\mathrm{OR}=1.84,95 \% \mathrm{Cl}=$ $1.51-2.24, p<0.001)$, after adjusting for transmission intensity. There was a borderline significant increased risk of malaria infection being undetectable by RDT among those who used an ITN and were microscopy positive (OR = $1.26,95 \% \mathrm{Cl}=1.00-1.59, p=0.053)$, whereas use of an ITN was associated with decreased RDT positivity $(\mathrm{OR}=0.84,95 \% \mathrm{Cl}=0.73-0.97, p=0.019)$ among microscopy-negative individuals. There was no evidence of an association between RDT brand and the risk of an undetected malaria infection by RDT among microscopy-positive individuals. Among microscopy-negative individuals, the proportion testing positive was different between RDT brands, but these results are difficult to interpret, owing to complete correlation between study and RDT brand. The year of the survey was not associated with discordant test results for RDT:microscopy.

Comparison of RDT- and PCR-measured prevalence. Analysis of RDT- and PCR-measured prevalence included 35,887 individuals tested with an RDT (cluster prevalence range $=0-45 \%$ ) and 31,178 individuals tested with PCR (cluster prevalence range $=0-52 \%$ ). There were a total of 162 geographical clusters across 17 countries (cluster size range $=5-3,307$, Figs 3a, b and Table 1). Pooled meta-analysis across all surveys showed that RDTs detected an average of $41 \%$ ( $95 \% \mathrm{Cl}=26-66 \%)$ of PCR-positive infections. This primarily reflects the relationship between RDT and PCR in low-transmission settings, with an average PCR prevalence of $8 \%$ across all the clusters included in our analysis.
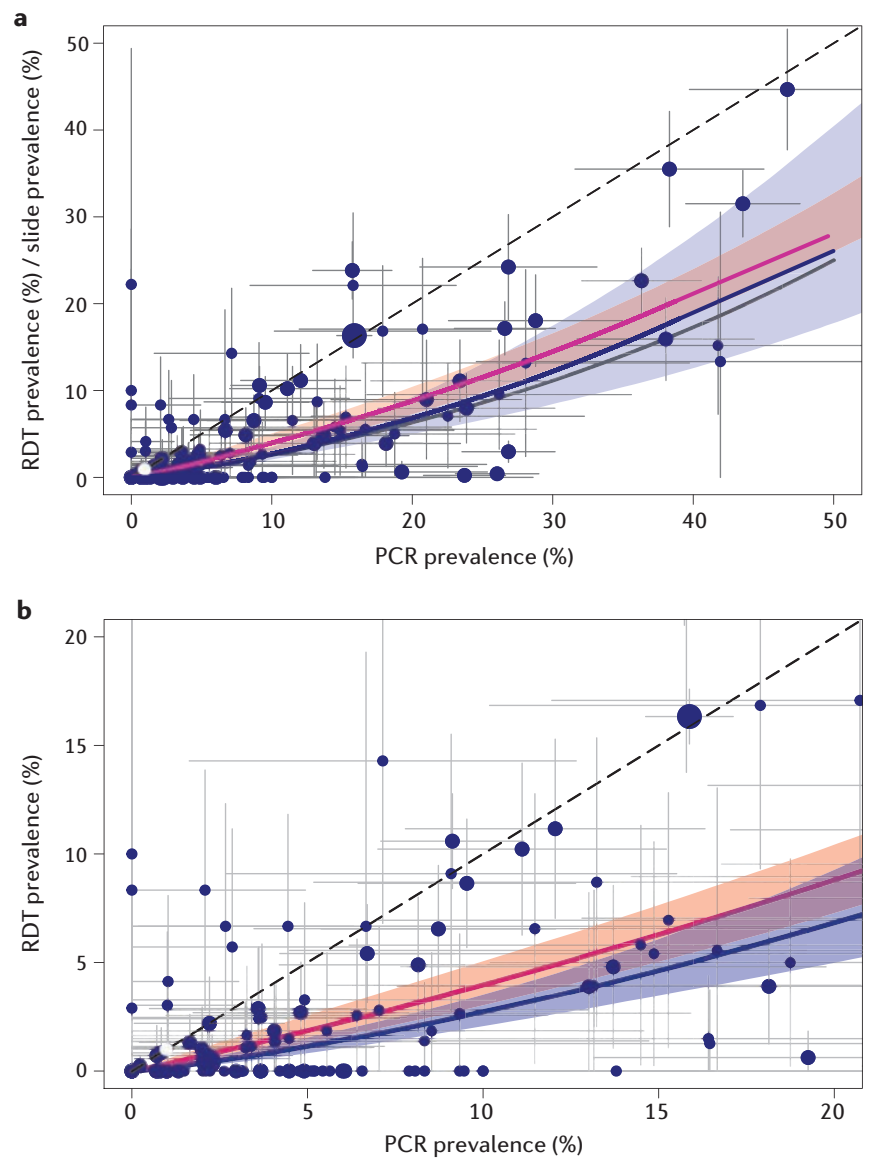

Figure 3 | The relationship between rapid diagnostic test (RDT) and polymerase chain reaction (PCR) prevalence overall (a) and zoomed in for $<20 \%$ PCR prevalence (b). Blue, observed RDT:PCR prevalence data and model fit; pink, the triangulated RDT:PCR comparison (see methods); grey, the PCR:microscopy comparison from ref. 13. Dashed lines indicate the expected relationship if RDT (or microscopy) and PCR detected equal prevalence. Horizontal and vertical lines indicate $95 \%$ confidence intervals around point estimates, whereas coloured solid lines indicate the median of the Bayesian posterior distributions from the fitted model and shaded areas indicate $95 \%$ credible intervals. Radius of point estimates indicate cluster size (from small to large: $<100,100-1,000$ and $>1,000$ ).

Age, transmission intensity and undetected malaria infection by RDT. As with the relationship between RDT- and microscopy-measured prevalence, stratifying by age group improved the model fit to the data, showing a decrease in detectability by RDT with increasing age (Figs 4a-c). Meta-analysis of the risk ratio between RDT and PCR positivity showed that, for children under 5 years of age, RDTs detected $81 \%$ ( $95 \% \mathrm{Cl}=74-89 \%)$ of PCR-positive infections. By comparison, RDTs detected fewer PCR-positive school-aged individuals ( $6-15$ years) $(70 \%, 95 \% \mathrm{Cl}=57-86 \%)$, and even fewer among adults over 15 years of age $(49 \%, 95 \% \mathrm{Cl}=31-78 \%)$. There was a larger data set available for analysis in the under 5 (140 clusters) and 6-15 (136 clusters) age groups compared with adults ( 81 clusters), suggesting that additional data in the higher age group could help to improve the accuracy of these estimates.

Previous studies have suggested that the proportion of carriers with sub-microscopic infections decreases in areas of higher transmission intensity, potentially because of an association with re-infection and increased parasite density ${ }^{13,14}$. A similar trend was also observed in the relationship between RDT and PCR detectability. The fit to our data was improved after stratifying by transmission intensity based on PCR-measured prevalence, showing increased RDT sensitivity compared with PCR as transmission increases (Fig. 4d-f). However, meta-analysis of the risk ratio between RDT and PCR positivity did not show a significant difference between the three transmission 
ranges, possibly indicating that more data are needed to define a more robust relationship for each transmission setting.

Figure 5 shows RDT detectability as a proportion of PCR-positive individuals, stratified by age and transmission intensity. Irrespective of transmission intensity, adults have the highest percentage of RDT-undetectable infections. By contrast, the percentage of individuals with RDT-detectable infections in all age groups increases as transmission intensity increases. However, since infection rates are greater at high-transmission intensities, RDTs may still miss a larger absolute number of infectious individuals at this level of endemicity. Best-fit model estimates of PCR-measured prevalence based on RDT-measured prevalence are summarized in Figs 3, 4 and Table 1.

Effect of individual-level covariates on RDT:PCR discordance. We evaluated the impact of age and transmission intensity on RDT positivity among PCR-negative individuals as a potential indicator of prolonged HRP2 clearance time. Logistic regression, adjusted for cluster PCR-measured prevalence, showed that among PCR-negative individuals, school-aged children had a significantly higher RDT positivity (OR $=1.53,95 \% \mathrm{Cl}=1.28-1.82, p<0.001)$ when compared with a baseline of children under 5 years of age. Adults showed similar odds of being RDT positive $(\mathrm{OR}=1.00,95 \% \mathrm{Cl}=0.64-1.58, p=0.990)$ as those under 5 years. Infections that were undetected by RDT, based on PCR positivity, were highest in adults $(\mathrm{OR}=5.04,95 \% \mathrm{Cl}=4.14-6.13, p<0.001)$ compared with those under 5 years, with a similar risk in school-aged children and those under 5 years (Supplementary Table 2 ).

RDT positivity among PCR-negative individuals varied between RDT brands, as did the detection of infection in PCR-positive individuals, but these results were not significant. Patients with a fever were less likely to have undetected infections by RDT if they were PCR positive $(\mathrm{OR}=0.14,95 \% \mathrm{Cl}=$ $0.06-0.32, p<0.001$ ), but also more likely to have a RDT-positive result if they were $P C R$ negative $(O R=4.86,95 \% \mathrm{Cl}=2.29-10.30, p<0.001)$. More recent surveys showed a lower risk of RDT-undetected infections, based on PCR positivity (OR $=0.77$ per year, $95 \% \mathrm{Cl}=0.60-0.99, p=0.044)$, which may indicate an improved performance of RDTs over time. PCR method was associated with test discordance at borderline significance, with RDTs detecting less PCR positive results measured by $\mathrm{QPCR}$ than those measured by PCR $(\mathrm{OR}=1.92$, $95 \% \mathrm{Cl}=0.98-3.74, p=0.056$ ), reflecting higher sensitivity of $\mathrm{QPCR}$, as described previously ${ }^{15,45}$.

Model validation. From the leave-one-out analysis, the correlation coefficient between observed and predicted values of RDT-measured prevalence from the RDT:PCR model was 0.67 , indicating a moderate agreement. The correlation coefficient between observed and predicted values of RDT-measured prevalence from the RDT:microscopy model was 0.92 , indicating a relatively stronger agreement (Fig. 6). The credible interval of this triangulated relationship was narrower than that of the directly observed line, owing to the larger number of data points in the RDT:microscopy and microscopy:PCR data sets (Figs 2, 3 , Table 1). There was no significant difference between the triangulated and observed relationships at any transmission intensity.

\section{DISCUSSION}

As the burden of malaria continues to decline in many regions ${ }^{1}$, it is crucial to understand the suitability of diagnostics for use in low-transmission and near-eliminating areas where MSAT and MDA strategies are likely to be applied. More specifically, how will diagnostic accuracy affect the ability of MSAT programmes to detect and treat asymptomatic individuals or determine local malaria prevalence thresholds for the initiation of MDA? Our study results show that the detection capability of RDTs is comparable with, and often greater than, microscopy. On average, microscopy captured $87 \%$ of RDT-positive infections, with higher test concordance in children than in adults. The extent to which this higher RDT detection reflects increased sensitivity, lack of specificity, or both, is unclear. Compared with molecular detection methods, however, RDTs still miss a substantial proportion of infections, capturing only $41 \%$ of PCR-positive individuals in low-transmission settings. Our analysis included cross-sectional data with paired prevalence measures by either RDT and microscopy or RDT and PCR from more than 180,000 individuals, spanning more than 400 geographical clusters. The detection levels a

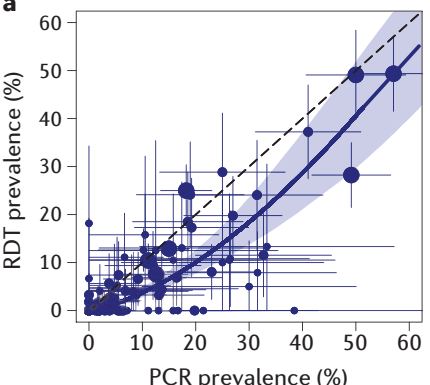

b

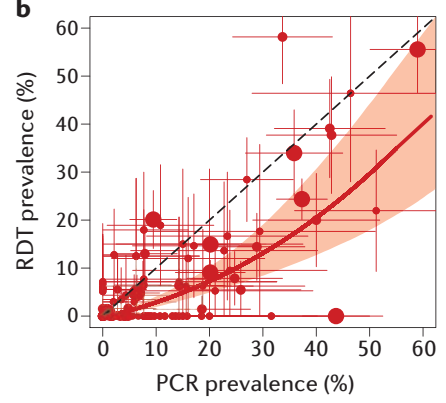

c

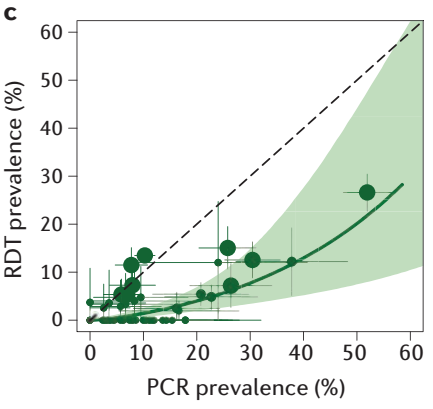

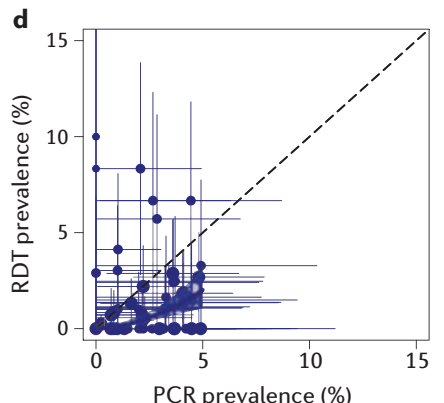

e
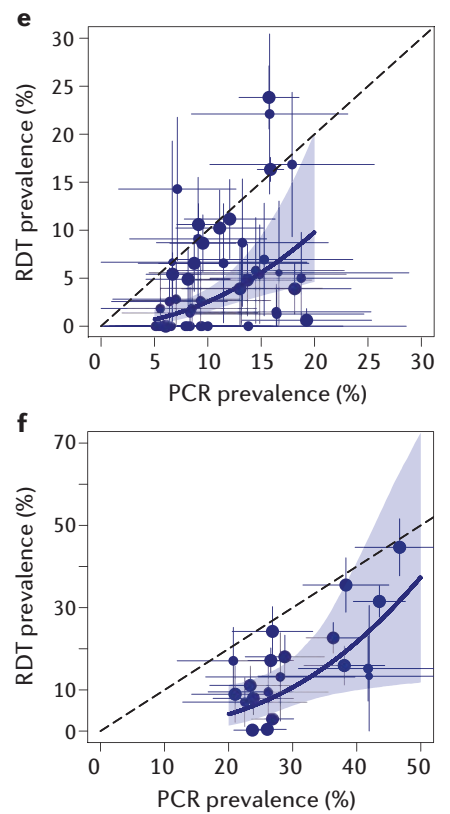

Figure 4 | The relationship between rapid diagnostic test (RDT) and polymerase chain reaction (PCR) prevalence by age group $(\mathrm{a}-\mathrm{c}$ ) and PCR prevalence band (d-f). The Bayesian model was fitted separately for each age group or PCR prevalence band. Age groups are younger than 5 years (a) $6-15$ years (b) and older than 15 years (c). PCR prevalence bands are $<5 \%$ (d), $5-20 \%$ (e) and $>20 \%$ (f). Dashed lines indicate the expected relationship if RDT and PCR detected equal prevalence. Horizontal and vertical lines around point estimates indicate $95 \%$ confidence intervals, whereas coloured solid lines indicate the median of the Bayesian posterior distributions from the fitted model and shaded areas indicate $95 \%$ credible intervals of these fits. Radius of point estimates indicate cluster size (from small to large: $<50,50-100$ and $>100$ ).

observed differed depending on age and transmission intensity, reflecting complex dynamics at both the ecological and host level that may influence parasite densities and the relative performance of these diagnostics.

Factors correlated with the accuracy of RDTs are varied and likely to be driven by subtleties in the concentration and duration of HRP2 antigens in peripheral circulation. A lower specificity by RDT is expected given that, in addition to current infection, they can detect recent infection owing to residual HRP2 even after parasite clearance. Our analysis found that RDTs had a higher positivity rate than microscopy among those who were more likely to have current or recent high parasite densities - children, those with measured or reported fever and those recently treated with antimalarial drugs. This may indicate that high parasite densities and, therefore, ruptured schizonts (asexual parasites that replicate to form multiple red blood cell invading parasites), lead to increased and/or prolonged HRP2 levels. These levels are likely to vary depending on an individual's clinical status and stage of infection owing to associated fluctuations in parasite density. Because RDTs have been designed for clinical use, it is intuitive that their performance would be optimal in the detection of high-density infections associated with symptomatic disease. A previous analysis evaluating the sensitivity of RDTs and microscopy, specifically in 


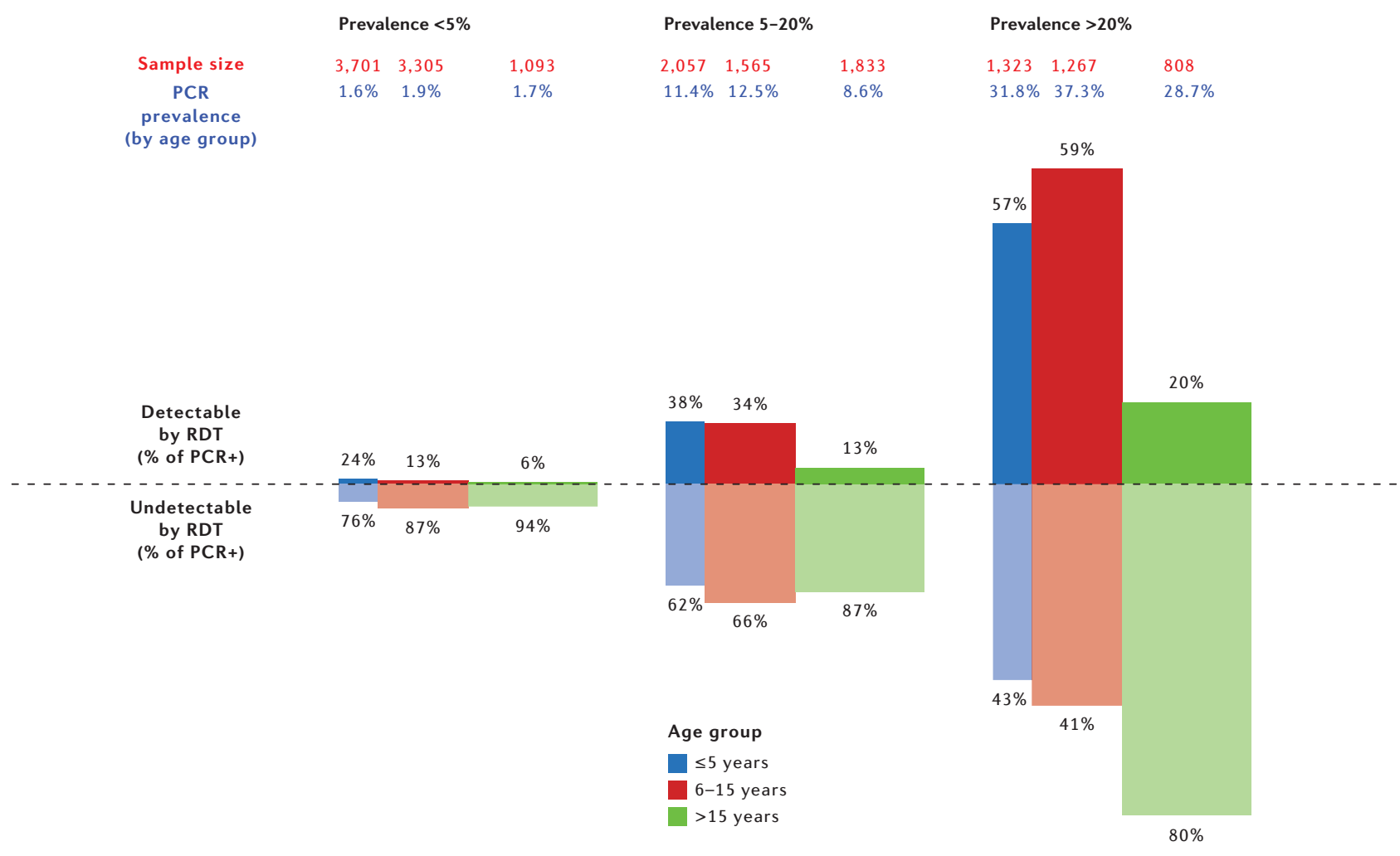

Figure 5 | Rapid diagnostic test (RDT) detectable (darker colours) and undetectable (lighter colours) infections based on polymerase chain reaction positive $(\mathrm{PCR}+$ ) infections by age (under 5 years, $6-15$ years and older than 15 years) and transmission intensity (PCR prevalence $<5 \%, 5-20 \%$ and $>20 \%$ ). The height of the bars for RDT detectable and undetectable proportions reflects the total prevalence of infection in that group according to PCR, whereas the width of the bars shows the proportion of the population in each age group in most African settings (younger than 5 years (blue), 15\%; 6-15 years (red), 35\%; and over 15 years (green), $50 \%$ of the total population ${ }^{70}$ ).

individuals with clinical symptoms, found an association between parasite density and RDT positivity ${ }^{60}$. This study also stressed the issue of false positives and how RDT specificity, in addition to being influenced by parasite density, may be correlated with age and transmission intensity. Further investigation into how RDT accuracy varies between clinical and subclinical populations could help to elucidate the factors that drive these differences. Our analysis also found that using an ITN was associated with better concordance of RDT and microscopy results, most probably due to a lower risk of infection. This distinction is particularly relevant for elimination strategies, because an RDT-positive and microscopy-negative result after parasite clearance may still indicate recent transmission in a population, whereas absence of infection does not. In general, it should be noted that the quality of microscopy is likely to vary more widely than that of RDTs. Microscopy in the context of research surveys is more accurate than those typically encountered during routine surveillance ${ }^{61}$. Therefore, the relative sensitivity of these diagnostics may be more discordant in programmatic settings than the relationship observed in this study.

Our analysis also found a number of factors that correlated with detection by RDT and PCR. Previous studies have demonstrated that the proportion of carriers with sub-microscopic infections decreases in areas of high-transmission intensity, potentially associated with superinfection (new malaria infection in already infected individuals $)^{13,14}$. This trend was also observed in our analysis - the proportion of PCR-measured infections that were detected by RDT increased with higher transmission intensity. Although the interaction between infection, immunity and parasite density in these settings is not fully understood, it has been suggested that only partial cross-immunity is acquired against malaria parasite clones ${ }^{62}$. Greater multiplicity of infection in higher transmission settings could result in higher parasite densities if host immune systems cannot respond to the diversity of parasites or if parasites increase growth rates in the presence of competing clones ${ }^{14,63}$. In addition to transmission intensity, we also observed age-associated variations in RDT detection. Our analysis shows that, after adjusting for transmission intensity, the odds of having an RDT-undetectable infection in adults was fivefold higher compared with under 5 year olds, potentially owing to more enhanced immune responses in adults that suppress parasite proliferation. This finding coincides well with data that show a lower sensitivity of microscopy relative to PCR among adults ${ }^{13}$. In addition, among PCR-positive individuals, the odds of a positive RDT result was seven times higher in patients with a fever. Overall, these results emphasize that fever, superinfections and childhood infections are commonly associated with high parasite densities, which, in turn, may lead to higher HRP2 levels that persist after parasite clearance. A number of studies have shown a relationship between parasite biomass and HRP2 clearance time ${ }^{64-66}$. However, these studies were predominantly in areas of high-density infections; studies in areas of lower parasite densities are less conclusive $^{61}$. Moreover, HRP2 concentrations may be influenced by duration of infection, parasite sequestration and HRP2 antibody responses ${ }^{67}$. Therefore, characterizing HRP2 detection profiles at parasite densities that are more typically found in elimination settings can help to better gauge the accuracy of RDTs in these areas. Our results also showed that risk of an RDT-positive and PCR-negative test result was higher in school-aged children compared with children under 5 and adults. This may be further evidence for an association between age and recent high parasite density (approximately 2-4 weeks), but may also suggest that infections can fall below the detection limit of PCR and still be captured by RDTs. RDT results that are typically presumed to be false positives may be advantageous when the identification of a recent as well as a current infection is needed, such as in elimination settings, or if HRP2 is still measurable during periods of fluctuating parasite density that drop below the molecular detection threshold. An improved understanding of RDT performance relative to PCR methods of various sensitivities, such as QPCR and LAMP, could help to further benchmark the range at which RDTs can optimally operate. Although the impact of the PCR method on test sensitivity has been investigated in previous studies ${ }^{14}$, more data are required to evaluate this relative to RDT sensitivity in more detail. 

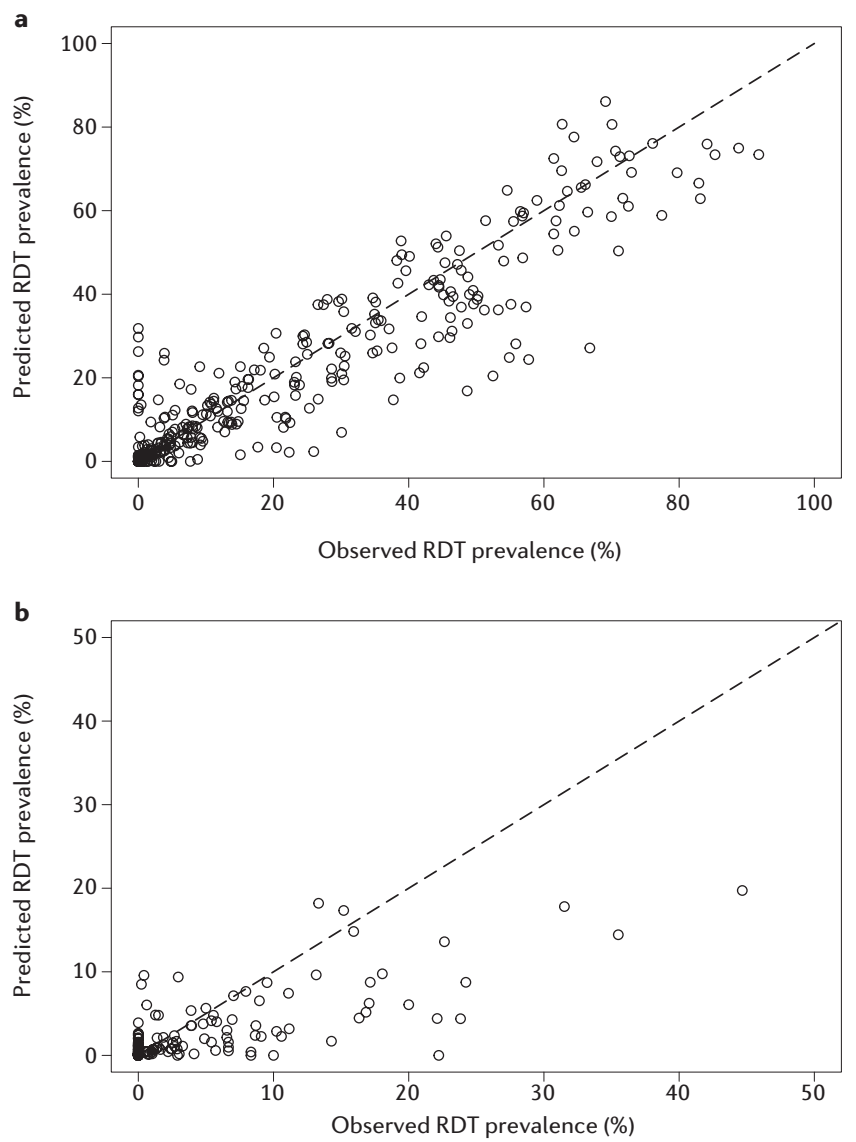

Figure 6 | The relationship between observed and predicted rapid diagnostic test (RDT) prevalence from the RDT:microscopy comparison (a), and the RDT:polymerase chain reaction comparison (b). Predictions were obtained using leave-one-out cross-validation.

We were able to define a more robust model for the relationship between prevalence measured by RDT compared with microscopy, than for the relationship between prevalence measured by RDT compared with PCR. This is because a more comprehensive data set of comparative RDT and microscopy measures was available across a wider range of transmission intensities. Medium- to high-transmission settings were particularly under-represented in the comparison of RDT and PCR measures. With more than half of our data from $<5 \%$ PCR prevalence settings (57\%; 93 of 162 clusters), the RDT:PCR relationship described here primarily reflects RDT performance at low-transmission intensity. However, the relationship between RDT- and PCR-measured prevalence estimated from directly observed paired data was not statistically different from the RDT:PCR relationship estimated by triangulating the RDT:microscopy and microscopy:PCR relationships based on independent data sets, improving confidence in our findings. Additional covariate information in future studies would further explain other factors that influence diagnostic sensitivity. Although we included RDT brand as a covariate in both the RDT:microscopy and RDT:PCR models, studies in this meta-analysis were not collected specifically to evaluate RDT brand so data are not sufficiently representative to draw conclusions on its impact on diagnostic sensitivity.

Overall, this study has established the relative detection capabilities of existing diagnostics for the identification of asymptomatic individuals infected with $P$. falciparum. To inform community chemotherapy programmes, however, further analysis is needed to determine to what extent these individuals contribute to onward transmission. As with detection, the potential infectiousness of asymptomatic individuals is sensitive to fluctuations in parasite density over the course of an infection and by season ${ }^{15,68}$. These are driven by the maturity of the host's immune response, which may vary by age and by local transmission dynamics, such as seasonality, that can influence population-level immunity or within-host parasite behaviour. Therefore, defining infectivity in relation to parasite density is especially important; this is addressed further by Slater and colleagues in a companion paper in this supplement ${ }^{69}$. Once the contribution of asymptomatic individuals to the infectious reservoir is better defined, future analyses should ideally establish optimal detection limits of new diagnostics for use in control and elimination strategies.

1. World Health Organization. World Malaria Report 2014 (WHO, 2014)

2. The malERA Consultative Group on Diagnoses and Diagnostics. A research agenda for malaria eradication: diagnoses and diagnostics. PLoS Med 8, e1000396 (2011).

3. Anthony, M. P., Burrows, J. N., Duparc, S., Moehrle, J. J. \& Wells, T. N. The global pipeline of new medicines for the control and elimination of malaria. Malar.J. 11, 316 (2012).

4. Tietje, K. et al. The essential role of infection-detection technologies for malaria elimination and eradication. Trends Parasitol. 30, 259-266 (2014).

5. Tiono, A. B. et al. Lessons learned from the use of HRP-2 based rapid diagnostic test in community-wide screening and treatment of asymptomatic carriers of Plasmodium falciparum in Burkina Faso. Malar. J. 13, 30 (2014).

6. Cook, J. et al. Mass screening and treatment using a falciparum-specific rapid diagnostic test did not reduce malaria incidence in Zanzibar. J. Infect. Dis. 211, jiu655 (2014).

7. Guerra, C. A. et al. Assembling a global database of malaria parasite prevalence for the Malaria Atlas Project. Malar. J. 6, 17 (2007).

8. Ochola, L. B., Vounatsou, P., Smith, T., Mabaso, M. L. H. \& Newton, C. R. J. C. The reliability of diagnostic techniques in the diagnosis and management of malaria in the absence of a gold standard. Lancet. Infect. Dis. 6, 582-588 (2006).

9. World Health Organization. Malaria Rapid Diagnostic Test Performance. Results of WHO Product Testing of Malaria RDTs: Round 4 (2012) (WHO, 2012).

10. Cordray, M. S. \& Richards-Kortum, R. R. Emerging nucleic acid-based tests for pointof-care detection of malaria. Am. J. Trop. Med. Hyg. 87, 223-230 (2012).

11. Andrews, L. et al. Quantitative real-time polymerase chain reaction for malaria diagnosis and its use in malaria vaccine clinical trials. Am. J. Trop. Med. Hyg. 73, 191-198 (2005).

12. Rockett, R. J. et al. A real-time, quantitative PCR method using hydrolysis probes for the monitoring of Plasmodium falciparum load in experimentally infected human volunteers. Malar. J. 10, 48 (2011).

13. Okell, L. C., Ghani, A. C., Lyons, E. \& Drakeley, C. J. Submicroscopic infection in Plasmodium falciparum-endemic populations: a systematic review and meta-analysis. J. Infect. Dis. 200, 1509-1517 (2009).

14. Okell, L. C. et al. Factors determining the occurrence of submicroscopic malaria infections and their relevance for control. Nature Commun. 3, 1237 (2012).

15. Bousema, T., Okell, L., Felger, I. \& Drakeley, C. Asymptomatic malaria infections: detectability, transmissibility and public health relevance. Nature Rev. Microbiol. 12, 833-840 (2014).

16. Stone, W., Gonçalves, B. P., Bousema, T. \& Drakeley, C. Assessing the infectious reservoir of falciparum malaria: past and future. Trends Parasitol. 31, 287-296 (2015)

17. Bousema, T. \& Drakeley, C. Epidemiology and infectivity of Plasmodium falciparum and Plasmodium vivax gametocytes in relation to malaria control and elimination. Clin. Microbiol. Rev. 24, 377-410 (2011).

18. Schneider, P. et al. Quantification of Plasmodium falciparum gametocytes in differential stages of development by quantitative nucleic acid sequence-based amplification. Mol. Biochem. Parasitol. 137, 35-41 (2004).

19. Dondorp, A. M. et al. Estimation of the total parasite biomass in acute falciparum malaria from plasma PfHRP2. PLoS Med 2, e204 (2005).

20. McMorrow, M. L., Aidoo, M. \& Kachur, S. P. Malaria rapid diagnostic tests in elimination settings--can they find the last parasite? Clin. Microbiol. Infect. Off. Publ. Eur. Soc. Clin. Microbiol. Infect. Dis. 17, 1624-1631 (2011).

21. Florey, L. Measures of Malaria Parasitemia Prevalence in National Surveys: Agreement Between Rapid Diagnostic Tests and Microscopy. DHS Analytical Studies No. 43. (2014).

22. Mouatcho, J. C. \& Goldring, J. P. D. Malaria rapid diagnostic tests: challenges and prospects. J. Med. Microbiol. 62, 1491-1505 (2013).

23. Endeshaw, T. et al. Evaluation of light microscopy and rapid diagnostic test for the detection of malaria under operational field conditions: a household survey in Ethiopia. Malar.J. 7, 118 (2008).

24. Falade, C. O. et al. Blood banking in a malaria-endemic area: evaluating the problem posed by malarial parasitaemias. Ann. Trop. Med. Parasitol. 103, 383-392 (2009).

25. Ganguly, S. et al. High prevalence of asymptomatic malaria in a tribal population in Eastern India. J. Clin. Microbiol. 51, 1439-1444 (2013).

26. Gitonga, C. W. et al. Use of rapid diagnostic tests in malaria school surveys in Kenya: does their under-performance matter for planning malaria control? Am. J. Trop. Med. Hyg. 87, 1004-1011 (2012).

27. Golassa, L., Enweji, N., Erko, B., Aseffa, A. \& Swedberg, G. Detection of a substantial number of sub-microscopic Plasmodium falciparum infections by polymerase chain reaction: a potential threat to malaria control and diagnosis in Ethiopia. Malar. J. 12, 352 (2013).

28. Gonçalves, L. et al. Bayesian latent class models in malaria diagnosis. PLoS ONE 7, e40633 (2012).

29. Ishengoma, D. S. et al. Accuracy of malaria rapid diagnostic tests in community studies and their impact on treatment of malaria in an area with declining malaria burden in north-eastern Tanzania. Malar.J. 10, 176 (2011).

30. Keating, J., Miller, J. M., Bennett, A., Moonga, H. B. \& Eisele, T. P. Plasmodium falciparum parasite infection prevalence from a household survey in Zambia using microscopy and a rapid diagnostic test: implications for monitoring and evaluation. Acta Trop. 112, 277-282 (2009). 
31. Laurent, A. et al. Performance of HRP-2 based rapid diagnostic test for malaria and its variation with age in an area of intense malaria transmission in southern Tanzania. Malar.J. 9, 294 (2010).

32. Mboera, L. E. G. et al. Comparison of the Paracheck-Pf test with microscopy, for the confirmation of Plasmodium falciparum malaria in Tanzania. Ann. Trop. Med. Parasitol. 100, 115-122 (2006).

33. Neumann, C. G. et al. Comparison of blood smear microscopy to a rapid diagnostic test for in-vitro testing for P. falciparum malaria in Kenyan school children. East Afr. Med.J. 85, 544-549 (2008).

34. Satoguina, J. et al. Comparison of surveillance methods applied to a situation of low malaria prevalence at rural sites in The Gambia and Guinea Bissau. Malar.J. 8, 274 (2009).

35. Shekalaghe, S. A. et al. Submicroscopic Plasmodium falciparum gametocyte carriage is common in an area of low and seasonal transmission in Tanzania. Trop. Med. Int. Heal. 12, 547-553 (2007)

36. Sousa-Figueiredo, J. C. et al. Investigating portable fluorescent microscopy (CyScope $\left.{ }^{\circledR}\right)$ as an alternative rapid diagnostic test for malaria in children and women of child-bearing age. Malar. J. 9, 245 (2010).

37. Wanji, S., Kimbi, H. K., Eyong, J. E., Tendongfor, N. \& Ndamukong, J. L. Performance and usefulness of the Hexagon rapid diagnostic test in children with asymptomatic malaria living in the Mount Cameroon region. Malar. J. 7, 89 (2008).

38. Ye, Y., Madise, N., Ndugwa, R., Ochola, S. \& Snow, R. W. Fever treatment in the absence of malaria transmission in an urban informal settlement in Nairobi, Kenya. Malar. J. 8, 160 (2009).

39. Dal-Bianco, M. P. et al. High prevalence of asymptomatic Plasmodium falciparum infection in Gabonese adults. Am. J. Trop. Med. Hyg. 77, 939-942 (2007).

40. Fancony, C., Sebastiao, Y., Pires, J., Gamboa, D. \& Nery, S. Performance of microscopy and RDTs in the context of a malaria prevalence survey in Angola: a comparison using PCR as the gold standard. Malar. J. 12, 284 (2013).

41. Ouattara, A. et al. Plasmodium falciparum infection and clinical indicators in relation to net coverage in central Cote d'Ivoire. Parasites Vectors 7, 306 (2014).

42. Faucher, J.-F. et al. What would PCR assessment change in the management of fevers in a malaria endemic area? A school-based study in Benin in children with and without fever. Malar. J. 9, 224 (2010).

43. ICF International, C. M. Demographic and Health Surveys (ICF, 2012).

44. Yeka, A. et al. Factors associated with malaria parasitemia, anemia and serological responses in a spectrum of epidemiological settings in Uganda. PLoS ONE 10, e0118901 (2015).

45. Mwingira, F., Genton, B., Kabanywanyi, A.-N. M. \& Felger, I. Comparison of detection methods to estimate asexual Plasmodium falciparum parasite prevalence and gametocyte carriage in a community survey in Tanzania. Malar. J. 13, (2014).

46. Harris, l. et al. A large proportion of asymptomatic Plasmodium infections with low and sub-microscopic parasite densities in the low transmission setting of Temotu Province, Solomon Islands: challenges for malaria diagnostics in an elimination setting. Malar. J. 9, 254 (2010)

47. Mharakurwa, S. et al. Pre-amplification methods for tracking low-grade Plasmodium falciparum populations during scaled-up interventions in Southern Zambia. Malar. J. 13, 89 (2014)

48. Tram, T. M. et al. An intensive longitudinal cohort study of Malian children and adults reveals no evidence of acquired immunity to Plasmodium falciparum infection. Clin. Infect. Dis. 57, 40-47 (2013)

49. Proietti, C. et al. Influence of infection on malaria-specific antibody dynamics in a cohort exposed to intense malaria transmission in northern Uganda. Parasite Immunol. 35, 164-173 (2013)

50. Stevenson, J. C. et al. Reliability of school surveys in estimating geographic variation in malaria transmission in the Western Kenyan Highlands. PLoS ONE 8, e77641 (2013).

51. Aydin-Schmidt, B. et al. Loop mediated isothermal amplification (LAMP) accurately detects malaria DNA from filter paper blood samples of low density parasitaemias. PLoS One 9, e103905 (2014).

52. Brown, T. et al. Molecular surveillance for drug-resistant Plasmodium falciparum in clinical and subclinical populations from three border regions of Burma/Myanmar: cross-sectional data and a systematic review of resistance studies. Malar. J. 11, 333

53. (28dR). .). et al. Loop-mediated isothermal amplification (LAMP) for point-of-care detection of asymptomatic low-density malaria parasite carriers in Zanzibar. Malar. J. 14, 43 (2015).

54. Stauffer, W. M. et al. Evaluation of malaria screening in newly arrived refugees to the United States by microscopy and rapid antigen capture enzyme assay. Pediatr. Infect. Dis. J. 25, 948-950 (2006).

55. Stresman, G. H. et al. A method of active case detection to target reservoirs of asymptomatic malaria and gametocyte carriers in a rural area in Southern Province, Zambia. Malar. J. 9, 265 (2010).
56. Sharp, S. J. \& Thompson, S. G. Analysing the relationship between treatment effect and underlying risk in meta-analysis: comparison and development of approaches. Stat. Med. 19, 3251-3274 (2000)

57. Hay, S. I., Smith, D. L. \& Snow, R. W. Measuring malaria endemicity from intense to interrupted transmission. Lancet. Infect. Dis. 8, 369-378 (2008).

58. World Health Organization. From Malaria Control to Malaria Elimination. A Manual for Elimination Scenario Planning (WHO, 2014).

59. Snounou, G. et al. High sensitivity of detection of human malaria parasites by the use of nested polymerase chain reaction. Mol. Biochem. Parasitol. 61, 315-320 (1993).

60. Abeku, T. A. et al. Determinants of the accuracy of rapid diagnostic tests in malaria case management: evidence from low and moderate transmission settings in the East African highlands. Malar. J. 7, (2008).

61. World Health Organisation. Parasitological Confirmation of Malaria Diagnosis. Report of a WHO Technical Consultation (WHO, 2010).

62. Ofosu-Okyere, A. et al. Novel Plasmodium falciparum clones and rising clone multiplicities are associated with the increase in malaria morbidity in Ghanaian children during the transition into the high transmission season. Parasitology 123, 113-23 (2001).

63. Pollitt, L. C. et al. Competition and the evolution of reproductive restraint in malaria parasites. Am. Nat. 177, 358-67 (2011).

64. Kyabayinze, D. J., Tibenderana, J. K., Odong, G. W., Rwakimari, J. B. \& Counihan, H. Operational accuracy and comparative persistent antigenicity of HRP2 rapid diagnostic tests for Plasmodium falciparum malaria in a hyperendemic region of Uganda. Malar. J. 7, 221 (2008).

65. Swarthout, T. D., Counihan, H., Senga, R. K. \& Broek, I. van den. Paracheck-Pf ${ }^{\circledR}$ accuracy and recently treated Plasmodium falciparum infections: is there a risk of over-diagnosis? Malar. J. 6, 58 (2007).

66. Houzé, S., Boly, M. D., Bras, J. Le, Deloron, P. \& Faucher, J.-F. PfHRP2 and PfLDH antigen detection for monitoring the efficacy of artemisinin-based combination therapy (ACT) in the treatment of uncomplicated falciparum malaria. Malar. J. 8, 211 (2009).

67. Aydin-Schmidt, B. et al. Usefulness of Plasmodium falciparum-specific rapid diagnostic tests for assessment of parasite clearance and detection of recurrent infections after artemisinin-based combination therapy. Malar. J. 12, 349 (2013).

68. Tusting, L. S., Bousema, T., Smith, D. L. \& Drakeley, C. Measuring changes in Plasmodium falciparum transmission: precision, accuracy and costs of metrics. Adv. Parasitol. 84, 151-208 (2014).

69. Slater, H. et al. Assessing the impact of next-generationrapid diagnostic testson Plasmodium falciparum malaria elimination strategies Nature 528, S94-S101 (2015).

70. United Nations, Department of Economic \& Social Affairs, Population Division. World Population Prospects, the 2010 Revision (UN, 2010).

SUPPLEMENTARY MATERIAL

Is linked to the online version of this paper at: http://dx.doi.org/10.1038/nature16039

\section{ACKNOWLEDGEMENTS}

We thank F. Mwingira and I. Felger (Tanzania), I. Harris and Q. Cheng (Solomon Islands), S. Portugal and P. Crompton (Mali), S. Mharakurwa and S. Volkman (Zambia), S.

Staedke and G. Dorsey (Uganda), C. Proietti and T. Bousema (Uganda), J. Stevenson, G. Stresman and J. Cox (Kenya), H. Kafy, A. Bashir, E. Malik, A. Mnzava, K. Subramaniam, K. Elmardi, I. Kleinschmidt and M. Donnelly (Sudan), M. Al-Selwei, S. Al-Eryani, A. Mnzava, A. Al-Samei, K. Mustafa, H. Atta, H. Al-Yarie, G. Zamanai and C. Barwa (Yemen) for kindly sharing their data from prevalence surveys, and the Demographic and Health Survey Programme for providing survey data. We would also like to thank L. Grignard, N. Alexander and J. Cook for sharing their expertise on statistical and diagnostic methods. This study was funded by the Bill and Melinda Gates Foundation (BMGF) Diagnostics Modelling Consortium. P.W. and L.O. acknowledge funding from fellowships jointly funded by the UK Medical Research Council (MRC) and the UK Department for International Development (DFID) under the MRC/DFID Concordat Agreement. L.W. acknowledges doctoral training funding from the UK MRC. A.G. acknowledges support from the BMGF, the Medicines for Malaria Venture, the UK MRC and the UK DFID. C.D. is funded by the Wellcome Trust grant number 091924.

COMPETING FINANCIAL INTERESTS

The authors declare no competing financial interests. Financial support for this publication has been provided by the Bill \& Melinda Gates Foundation.

\section{ADDITIONAL INFORMATION}

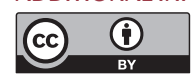

This work is licensed under the Creative Commons Attribution 4.0 International License. The images or other third party material in this article are included in the article's Creative Commons license, unless indicated otherwise in the credit line; if the material is not included under the Creative Commons license, users will need to obtain permission from the license holder to reproduce the material. To view a copy of this license, visit http://creativecommons.org/licenses/by/4.0 